\title{
In vitro evaluation of cytotoxic properties of 5-Aminolevulinic acid (5-ALA) on bladder cancer cells
}

\begin{abstract}
Introduction: 5-Aminolevulinic (5-ALA) may be used as a photo diagnostic agent in bladder cancer. The aim of this study was to investigate the cytotoxic effect of 5-ALA on bladder cancer cells. Materials and methods: T24 cells treated with various concentrations of mitomycin (MC), 5-ALA and an MC/5-ALA mixture were evaluated to determine the role of 5-ALA on MC cytotoxicity. Cell cycle analysis was conducted, and apoptosis was analyzed by flow cytometry. Caspase 3 enzyme and reactive oxygen species were measured. Results: Our initial studies exploring the impact of combination therapy on cell viability demonstrated improved cytotoxic effects on T24 and RT cells with relatively low doses of 5-ALA/MC in conjunction with MC alone. Indicated no significant difference between the IC50 of MC and MC/5-ALA in T24 cells, while IC50 value was decreased by $25 \%$ in RT4 cells in 5-ALA/MC in comparing with $\mathrm{MC}$ alone. However, examination of cell cycle phase arrests by flow cytometry revealed significant PreG1 apoptosis and cell growth arrest in G2/M in T24 cells treated with the MC/5ALA mixture compared with MC treatment. In addition, caspase 3 enzyme was increased by 1.15-fold in T24 cells treated with MC/5-ALA in comparison with MC alone. Conclusion: These results suggest that 5-ALA might possess anti-cancer properties and is not only a photo diagnostic element.
\end{abstract}

Keyword: Cytotoxicity; Bladder cancer; 5-Aminolevulinic acid; Hexaminolevulinate; Chemotherapy; Apoptosis 